\title{
Análisis comparativo de escalas diagnósticas de apendicitis aguda: Alvarado, RIPASA y AIR
}

\author{
Comparative analysis of diagnostic scales of acute appendicitis: Alvarado, RIPASA \\ and AIR
}

\author{
Martín A. Bolívar-Rodríguez ${ }^{*}$, Benny A. Osuna-Wong ${ }^{1}$, Ana B. Calderón-Alvarado', Jaime Matus-Rojas ${ }^{1}$, \\ Edgar Dehesa-López y Felipe de Jesús Peraza-Garay ${ }^{3}$ \\ ${ }^{1}$ Departamento de Cirugía General; ${ }^{2}$ Departamento de Investigación; ${ }^{3}$ Departamento de Estadística. Centro de Investigación y Docencia en Ciencias \\ de la Salud, Universidad Autónoma de Sinaloa, Culiacán, Sin. México
}

\section{Resumen}

Introducción: La apendicitis aguda es la enfermedad quirúrgica más común en cirugía de urgencia; sin embargo, sigue siendo un problema diagnóstico y representa un reto a pesar de la experiencia y los diferentes métodos de diagnóstico clínicos y paraclínicos. Objetivo: Evaluar en forma comparativa las escalas de Alvarado, AIR y RIPASA para determinar cuál es superior como prueba diagnóstica de apendicitis aguda en nuestra población, llegando a un diagnóstico preciso en el menor tiempo y costo posibles. Método: Estudio observacional, prospectivo, transversal y comparativo de 137 pacientes a quienes se aplicó las escalas de Alvarado, AIR y RIPASA, que ingresaron al servicio de urgencias del Hospital Civil de Culiacán (México) con síndrome doloroso abdominal sugestivo de apendicitis aguda. Resultados: La escala de Alvarado presentó una sensibilidad del 97.2\% y una especificidad del 27.6\%. AIR tuvo una sensibilidad del $81.9 \%$ y una especificidad del 89.5\%. RIPASA arrojó los mismos resultados que Alvarado. Todas las pruebas tuvieron una exactitud diagnóstica por arriba del $80 \%$. Conclusiones: Alvarado y RIPASA presentaron buena sensibilidad, mientras que AIR es más específica y tiene mayor exactitud diagnóstica de apendicitis aguda, realizando un mejor tamizaje y permitiendo disminuir las cirugías innecesarias, por lo que se recomienda usar más AIR que Alvarado y RIPASA.

PALABRAS CLAVE: Apendicitis. Diagnóstico. Alvarado. RIPASA. AIR.

\begin{abstract}
Introduction: Acute appendicitis is the most common surgical disease in emergency surgery, however, it remains a diagnostic problem and represents a challenge despite the experience and the different clinical and paraclinical diagnostic methods. Objective: To evaluate in a comparative way the scale of Alvarado, AIR and RIPASA to determine which one is best as a diagnostic test of acute appendicitis in our population in order to arrive to an accurate diagnosis in the shortest possible time and cost. Method: Observational, prospective, transversal and comparative study of 137 patients to whom the scale of Alvarado, AIR and RIPASA was applied, who entered the emergency service of the Civil Hospital of Culiacán (México) with abdominal pain syndrome suggestive of acute appendicitis. Results: The Alvarado scale presented sensitivity $97.2 \%$ and specificity of $27.6 \%$. AlR presented sensitivity of $81.9 \%$ and specificity of $89.5 \%$. RIPASA showed the same results as Alvarado. All tests showed diagnostic accuracy above $80 \%$. Conclusions: Alvarado and RIPASA presented good sensitivity, however, AIR is more specific, and has better accuracy for the diagnosis of acute appendicitis, making a better screening and thus reducing unnecessary surgeries. Therefore, it is recommended to use more AIR than Alvarado and RIPASA.
\end{abstract}

KEY WORDS: Appendicitis. Diagnosis. Alvarado. RIPASA. AIR.

\footnotetext{
Correspondencia:

*Martín A. Bolívar Rodríguez

Eustaquio Buelna 91,

Col. Gabriel Leyva

C.P. 80030 Culiacán, Sin., México

E-mail: bolivarmartin64@ hotmail.com
}

Fecha de recepción: 02-02-2018

Fecha de aceptación: 16-04-2018

DOI: 10.24875/CIRU.M18000029
Cir Cir. 2018;86:169-174

Contents available at PubMed www.cirugiaycirujanos.com 


\section{Introducción}

La apendicitis aguda es la enfermedad quirúrgica que con más frecuencia causa hospitalización y cirugía de emergencia; sin embargo, aun después de un lapso tan prolongado de tiempo desde su primera descripción, sigue siendo en ocasiones un problema de diagnóstico y representa un reto para todos los médicos que atienden al paciente con sintomatología sugestiva de este proceso patológico, a pesar de la experiencia y de los diferentes métodos de diagnóstico clínicos y paraclínicos.

La apendicectomía es actualmente el procedimiento quirúrgico de urgencia más común en el mundo. El riesgo de presentar apendicitis aguda a lo largo de la vida se calcula en aproximadamente un $7 \%$. Su incidencia es de 11 casos por cada 10,000 personas al año en los países desarrollados'. En los EE.UU. se reportan anualmente 250,000 casos de apendicitis, que representan un millón de días de hospitalización-paciente al año ${ }^{2}$. En nuestra población se estima que una de cada 15-20 personas presentará apendicitis aguda en algún momento de su vida, reportando una incidencia de 1.51.9 casos por cada 1000 habitantes $^{3}$.

El diagnóstico incorrecto o tardío aumenta el riesgo de complicaciones, y por ende la tasa de diagnósticos erróneos de apendicitis se reportó entre un 15 y un $25 \%$, considerándose aceptable para reducir la incidencia de complicaciones ${ }^{4}$. Sin embargo, en los últimos años la frecuencia de apendicectomías negativas ha ido disminuyendo de manera mantenida en relación con el uso de estudios diagnósticos de imagen. "Ahora hay clara evidencia de que (los rangos usados previamente) no deben seguir siendo considerados aceptables» ${ }^{5}$. Sin embargo, en nuestro medio, la historia médica de rutina y la exploración física continúan siendo las modalidades diagnósticas más prácticas ${ }^{6}$. Hoy por hoy, un sinnúmero de autores aseveran que el diagnóstico de apendicitis aguda continúa siendo clínico, como refieren Rebollar, et al.7: «una historia clínica bien desarrollada, así como una buena exploración física, dan el diagnóstico en la mayoría de los casos".

A pesar de ser una afección común, sigue siendo un diagnóstico difícil de establecer, en especial en los grupos de población de jóvenes, ancianos y mujeres en edad reproductiva, en las que diversas condiciones inflamatorias del tracto ginecológico y genitourinario pueden presentar signos y síntomas que son similares a los de la apendicitis aguda. Un retraso en la realización de la apendicectomía con el fin de mejorar su precisión diagnóstica aumenta el riesgo de perforación apendicular y sepsis, así como la morbilidad y la mortalidad 8 .

Actualmente existen numerosas herramientas que pueden utilizarse en la evaluación de los pacientes con sospecha de apendicitis. Cómo estas herramientas son combinadas en la práctica depende en gran medida del marco, la disponibilidad de recursos y los objetivos clínicos. Por ejemplo, en zonas rurales de Kenia, la evaluación clínica sigue siendo la piedra angular del diagnóstico para todos. En Washington, los objetivos de diagnóstico varían dependiendo del paciente: para los niños, minimizar la exposición a radiación ionizante puede mitigar el deseo de mayor información diagnóstica; para las mujeres de 18 a 35 años de edad, precisamente descartar una enfermedad no apendicular puede ser una prioridad; para pacientes ancianos, en quienes la radiación es de menos riesgo, la claridad y la cantidad de información proporcionada por la tomografía computarizada pueden justificar su uso sistemático ${ }^{9}$.

Por ende, existen diversas escalas (Alvarado, RIPASA y AIR) que incluyen los signos y síntomas clásicos de la apendicitis aguda más los estudios de laboratorio.

La escala de Alvarado (Tabla 1) es la más conocida y la que hasta hace unos años mostraba el mejor rendimiento en estudios de validación ${ }^{10}$. Fue desarrollada en 1986 por el Dr. Alfredo Alvarado"11. La escala AIR (Appendicitis Inflammatory Response) (Tabla 2) es un sistema de puntuación, creado en Suecia en el año 2008, desarrollado por Andersson y Andersson ${ }^{12}$. Más recientemente se ha dado a conocer la escala RIPASA (Tabla 3) en el Hospital Raja Isteri Pengiran Anak Saleha (RIPAS), por Chong, et al. ${ }^{13}$; esta escala fue elaborada en Asia en el año 2010, por lo que su aplicabilidad y efectividad en otras poblaciones sigue en estudio.

\section{Método}

Se realizó un estudio observacional, prospectivo, transversal y comparativo de 137 pacientes a quienes se aplicaron las escalas de Alvarado, AIR y RIPASA, que ingresaron al servicio de urgencias del Hospital Civil de Culiacán con síndrome doloroso abdominal sugestivo de apendicitis aguda. 
Tabla 3. Escala RIPASA

\begin{tabular}{lc}
\hline Escala RIPASA & Puntos \\
\hline Datos & 1 \\
Hombre & 0.5 \\
Mujer & 1 \\
$<39.9$ años & 0.5 \\
$>40$ años & 1 \\
Extranjero & \\
Síntomas & \\
Dolor en fosa iliaca derecha & 0.5 \\
Náuseas/vómito & 1 \\
Dolor migratorio & 0.5 \\
Anorexia & 1 \\
Síntomas < 48 horas & 1 \\
Síntomas > 48 horas & 0.5 \\
Signos & \\
Hipersensibilidad en fosa iliaca derecha & \\
Resistencia muscular voluntaria & 1 \\
Rebote & 2 \\
Rovsing & 1 \\
Fiebre $>37$ y $<39^{\circ} \mathrm{C}$ & 2 \\
Laboratorio & 1 \\
Leucocitosis & \\
Análisis general de orina negativo & 1 \\
\hline
\end{tabular}

Improbable: menos de 5.0 puntos. Observación del paciente en hospitalización temporal y revalorar en 1 a 2 horas. Si disminuye el puntaje, egreso; si aumenta, tratar de acuerdo con el puntaje.

Baja probabilidad: 5.0-7.0 puntos. Observación en hospitalización temporal y revalorar en 1 a 2 horas, o realizar estudio de imagen para descartar apendicitis aguda.

Alta probabilidad: 7.5-11.0 puntos. Interconsulta a cirugía y admisión. Revalorar en a 2 horas. Si permanece alto, preparar al paciente para apendicectomía. En mujeres, sugerir realización de ultrasonografía para descartar causa ginecológica.

Diagnóstico de apendicitis aguda: más de 12 puntos. Referir a cirugía para realización de apendicectomía.

negativo en 10 (7.29\%); y 7 (5.10\%) para riesgo bajo, de los cuales el hallazgo histopatológico fue positivo en $4(2.91 \%)$ y negativo en $3(2.18 \%)$. Recopilando todos los datos, se obtuvieron $105(76.64 \%)$ verdaderos positivos, 21 (15.32\%) pacientes con escala positiva y biopsia negativa, 8 (5.83) verdaderos negativos y $3(2.18 \%)$ pacientes con escala negativa y biopsia positiva. Así, esta escala diagnóstica muestra una sensibilidad del $97.2 \%$, una especificidad del $27.6 \%$, un valor predictivo positivo del $83.3 \%$, un valor predictivo negativo del $72.7 \%$ y una exactitud diagnóstica del $82.5 \%$.

El grupo RIPASA estuvo compuesto por 137 pacientes a los cuales se aplicó la escala diagnóstica y se obtuvo un total de 68 (49.63\%) pacientes con alta probabilidad, de los cuales el hallazgo histopatológico fue positivo en 53 (38.68\%) y negativo en $15(10.94 \%)$; hubo 9 (6.56\%) pacientes para baja probabilidad, de los cuales el hallazgo histopatológico fue positivo en $2(1.45 \%)$ y negativo en 7 (5.10\%); de 58 (42.33\%) pacientes en el rubro de diagnóstico, el estudio histopatológico fue positivo en 52 (37.95\%) y negativo en
6 (4.37\%); y de 2 pacientes en el rubro de improbable, $1(0.72 \%)$ obtuvo resultado histopatológico positivo y $1(0.72 \%)$ negativo. Recopilando todos los datos, hubo 105 (76.64\%) verdaderos positivos, 21 (15.32\%) pacientes con escala positiva y estudio histopatológico negativo, 8 (5.83\%) verdaderos negativos y $3(2.18 \%)$ con escala negativa y estudio histopatológico positivo. Así, esta escala diagnóstica muestra una sensibilidad del $97.2 \%$, una especificidad del $27.6 \%$, un valor predictivo positivo del $83.3 \%$, un valor predictivo negativo del $72.7 \%$ y una exactitud diagnóstica del $82.5 \%$.

El grupo AIR estuvo compuesto por 137 pacientes, de los cuales se eliminaron 48 al no cumplir con los parámetros requeridos para la escala diagnóstica. Finalmente se incluyeron 91 pacientes, a los que se aplicó la escala diagnóstica y se obtuvo un total de $13(9.48 \%)$ con alta probabilidad, de los cuales el hallazgo histopatológico fue positivo en 11 (8.02\%) y negativo en 2 (1.45\%); de 24 (17.51\%) para baja probabilidad, el estudio histopatológico fue positivo en $13(9.48 \%)$ y negativo en 11 (8.02\%); y de $52(37.95 \%)$ con riesgo intermedio, el estudio histopatológico fue positivo en 46 (33.57\%) y negativo en 6 (4.37\%). Recopilando todos los datos, hubo 59 (64.83\%) verdaderos positivos, 2 (2.19\%) pacientes con escala positiva y estudio histopatológico negativo, 17 (18.68\%) verdaderos negativos y $13(14.28 \%)$ pacientes con escala negativa y estudio histopatológico positivo. Así, esta escala diagnóstica muestra una sensibilidad del $81.9 \%$, una especificidad del $89.5 \%$, un valor predictivo positivo del $96.7 \%$, un valor predictivo negativo del $56.7 \%$, una proporción de falsos positivos del $10.5 \%$, una proporción de falsos negativos del $18.1 \%$ y una exactitud diagnóstica del $83.5 \%$ (Tabla 4).

\section{Discusión}

Como ya se describió, en nuestra revisión no se encontró en la literatura mundial ningún estudio que realice una comparación de estas tres escalas diagnósticas, pero sí numerosos que comparan Alvarado y AIR, o Alvarado y RIPASA. Comparando nuestros resultados con los disponibles en la literatura mundial, encontramos que en los estudios de Andersson y Andersson ${ }^{12}$ en cuanto a AIR y Alvarado se refiere existe un completo contraste, pero el estudio de Sammalkorpi, et al. ${ }^{14}$ muestra similitud con el nuestro en cuanto a especificidad, con una mejor sensibilidad para el nuestro, comparando AIR y Alvarado. En cuanto a los estudios realizados por Reyes-García, 
Tabla 4. Resultados de las escalas diagnósticas

\begin{tabular}{|c|c|c|c|c|c|c|}
\hline \multicolumn{7}{|l|}{ Resultados } \\
\hline & Alvarado, $\%$ & IC, \% & $\begin{array}{c}\text { RIPASA, } \\
\%\end{array}$ & $\begin{array}{l}\text { IC, } \\
\%\end{array}$ & $\begin{array}{c}\text { AIR, } \\
\%\end{array}$ & IC, $\%$ \\
\hline Sensibilidad & 97.2 & $\begin{array}{l}92.1 \\
99.1\end{array}$ & 97.2 & $\begin{array}{l}92.1 \\
99.1\end{array}$ & 91.9 & $\begin{array}{l}71.5 \\
89.1\end{array}$ \\
\hline Especificidad & 27.6 & $\begin{array}{l}14.7 \\
45.7\end{array}$ & 27.6 & $\begin{array}{l}14.7 \\
45.7\end{array}$ & 89.5 & $\begin{array}{l}68.6 \\
97.1\end{array}$ \\
\hline VPP & 83.3 & $\begin{array}{l}75.9 \\
88.8\end{array}$ & 83.3 & $\begin{array}{l}75.9 \\
88.8\end{array}$ & 96.7 & $\begin{array}{l}88.8 \\
99.1\end{array}$ \\
\hline VPN & 72.7 & $\begin{array}{l}43.4 \\
90.3\end{array}$ & 72.7 & $\begin{array}{l}43.4 \\
90.3\end{array}$ & 56.7 & $\begin{array}{l}39.2 \\
72.6\end{array}$ \\
\hline PFP & 72.4 & $\begin{array}{l}54.3 \\
85.3\end{array}$ & 72.4 & $\begin{array}{l}54.3 \\
85.3\end{array}$ & 10.5 & $\begin{array}{r}2.9 \\
31.4\end{array}$ \\
\hline PFN & 2.8 & $\begin{array}{l}0.9 \\
7.9\end{array}$ & 2.8 & $\begin{array}{l}0.9 \\
7.9\end{array}$ & 18.1 & $\begin{array}{l}10.9 \\
28.5\end{array}$ \\
\hline Exactitud & 82.5 & $\begin{array}{l}75.3 \\
87.9\end{array}$ & 82.5 & $\begin{array}{l}75.3 \\
87.9\end{array}$ & 83.5 & $\begin{array}{l}74.6 \\
89.7\end{array}$ \\
\hline
\end{tabular}

IC: intervalo de confianza; PFN: proporción de falsos negativos; PFP: proporción de

falsos positivos; VPN: valor predictivo negativo; VPP: valor predictivo positivo.

et al. ${ }^{15}$ en 2012, Nanjundaiah, et al. ${ }^{8}$ en 2014 , y Walczak DA, et al. ${ }^{16}$ en 2015, presentan resultados similares a los encontrados para la escala RIPASA en el presente estudio, compartiendo resultados con la literatura mundial.

El primer estudio publicado al respecto se realizó en Suecia en el año 2008, y en él los autores Andersson y Andersson ${ }^{12}$ obtuvieron una precisión diagnóstica del $93 \%$ para apendicitis avanzada y del $97 \%$ para todas las apendicitis, frente al $92 \%(p=0.0027)$ y el $88 \%(p=0.0007)$, respectivamente, para la escala de Alvarado.

En cuanto a exactitud diagnóstica se refiere, se obtuvo un $82.5 \%$ de precisión diagnóstica para Alvarado y RIPASA, frente a un $83.5 \%$ para AIR, teniendo similares valores a los disponibles en la literatura.

Burgos-Oliveros ${ }^{17}$ realizó un estudio con el objetivo de evaluar las diferencias entre estas escalas, que incluyó 352 pacientes de 14 a 60 años de edad con diagnóstico de apendicitis aguda, a quienes se efectuó una apendicectomía. La escala RIPASA muestra superioridad frente a la escala de Alvarado; en nuestros resultados, la escala de Alvarado y RIPASA comparten los mismos valores diagnósticos. En el mismo tema, Walczak, et al. ${ }^{16}$ hicieron un estudio que incluyó 92 pacientes a los que se realizó laparotomía bajo sospecha de apendicitis aguda. En este trabajo se comparan cinco escalas distintas, entre ellas RIPASA y Alvarado. Concluyen que por los hallazgos de los sistemas de escalas tienen un valor limitado para el diagnóstico de apendicitis aguda. La mayoría de los sistemas utilizados mostraron alta sensibilidad y valor predictivo positivo, permitiendo seleccionar a los pacientes verdaderamente enfermos y disminuir las laparotomías innecesarias. Por otra parte, se evidencian una especificidad y valor predictivo negativo bajos, lo que podría asociarse a diagnóstico tardío y las subsecuentes complicaciones. Estos resultados difieren de los del resto de la literatura, particularmente en lo concerniente a la especificidad. Nuestro valor predictivo positivo fue alto para las tres escalas, con valores del $83.3 \%$ para Alvarado y RIPASA, y del $96.7 \%$ para AIR; los valores predictivos negativos fueron bajos.

\section{Conclusiones}

Concluimos en el presente estudio que la escala de Alvarado y la escala RIPASA tienen un muy buen margen para detectar pacientes verdaderamente enfermos cuando presentan alto riesgo, contando con una sensibilidad del $97.2 \%$, y un alto valor predictivo positivo del $83.3 \%$, teniendo una precisión diagnóstica que alcanza el $82.5 \%$.

En cuanto a la escala AIR, presenta una adecuada especificidad del $89.5 \%$ y una sensibilidad del $81.9 \%$ cuando encasilla a los pacientes en alta probabilidad y probabilidad intermedia, con un valor predictivo positivo que alcanza hasta el $96.7 \%$, y una exactitud diagnóstica del $83.5 \%$.

Sin embargo, la pesquisa para el diagnóstico prequirúrgico del paciente con sospecha de apendicitis aguda continúa siendo desafiante, incluso después de la introducción de estudios de gabinete, por lo que estas herramientas deben ser utilizadas de manera selectiva. Aunque su diagnóstico se considera relativamente fácil, los signos clásicos de apendicitis a veces pueden ser difíciles de obtener y solo están presentes inequívocamente en el $50 \%$ de los pacientes.

Este estudio buscó determinar qué escala tiene una mayor precisión como prueba diagnóstica, obteniendo más precisión la escala AIR. Esta puede ser aplicada a nuestra población como un método objetivo que respalde la toma de decisiones en el manejo de un paciente con sospecha de apendicitis aguda.

En cuanto a la tasa de apendicectomías negativas se refiere, creemos firmemente que estamos en un 
rango aceptable (13.6\%) y que nos enfocaremos más en el seguimiento a corto plazo de los pacientes con sospecha.

\section{Conflicto de intereses}

Los autores declaran no tener conflicto de intereses.

\section{Bibliografía}

1. Petroianu A. Diagnosis of acute appendicitis. Int J Surg. 2012;10:115-9

2. Craig S. Appendicitis. Medscape. (Actualizado el 21 de julio de 2014; consultado el 10 de diciembre de 2015.) Disponible en: http://emedicine. medscape.com/article/773895-overview\#a6.

3. Asociación Mexicana de Cirugía General A.C. Guía de práctica clínica apendicitis aguda. México D.F; 2014. (Consultado el 10 de diciembre de 2015.) Disponible en: https://amcg.org.mx/images/guiasclinicas/apendicitis.pdf.

4. Shogilev DJ, Duus N, Odom SR, Shapiro NI. Diagnosing appendicitis: evidence-based review of the diagnostic approach in 2014. West Emerg Med. 2014;15:859-71.

5. Drake FT, Flum DR. Improvement in the diagnosis of appendicitis. Adv Surg. 2013;47:299-328

6. Wagner J, Mckinney P, Carpenter J. Does this adult patient have appendicitis? JAMA. 1996;276:1589-94.
7. Rebollar González RC, García Álvarez J, Trejo Téllez R. Apendicitis aguda: revisión de la literatura. Rev Hosp Jua Mex. 2009;76:210-6.

8. Nanjundaiah N, Ashfaque M, Venkatesh S, Kalpana A, Priya SA. A comparative study of RIPASA score and Alvarado score in the diagnosis of appendicitis. J Clin Diagn Res. 2014;8:NC03-5.

9. Thurston Drake F, Reed Flum D. Improvement in the diagnosis of appendicitis. Adv Surg. 2013;47:299-328.

10. Ohmann C, Yang Q, Franke C. Diagnostic scores for acute appendicitis. Abdominal Pain Study Group. Eur J Surg. 1995;161:273-81.

11. Alvarado A. A practical score for the early diagnosis of acute appendicitis. Ann Emerg Med. 1986;15:557-64.

12. Andersson M, Andersson RE. The Appendicitis Inflammatory Response score: a tool for the diagnosis of acute appendicitis that outperforms the Alvarado score. World J Surg. 2008;32:1843-9.

13. Chong CF, Thien A, Mackie AJA, Tin AS, Tripathi S, Ahmad MA et al. Comparison of RIPASA and Alvarado scores for the diagnosis of acute appendicitis. Singapore Med J. 2011;52:340-5.

14. Sammalkorpi HE, Mentula $P$, Leppäniemi A. A new adult appendicitis score improves diagnostic accuracy of acute appendicitis - a prospective study. BMC Gastroenterol. 2014;14:114.

15. Reyes-García N, Zaldívar-Ramírez FR, Cruz-Martínez R, Sandoval-Martínez MD, Gutiérrez-Banda CA, Athié-Gutiérrez C. Precisión diagnóstica de la escala RIPASA para el diagnóstico de apendicitis aguda: análisis comparativo con la escala de Alvarado modificada. Cir Gen. 2012; 34:101-6.

16. Walczak DA, Pawelczak D, Zoltaszek A, Jaguscik R, Falek W, Czerwinska M, et al. The value of scoring systems for the diagnosis of acute appendicitis. Pol Przegl Chir. 2015;87:65-70.

17. Burgos-Oliveros HB. Comparación entre los scores de RIPASA y Alvarado en el diagnóstico de apendicitis aguda. Tesis de titulación. Universidad Privada Antenor Orrego; 2014. 